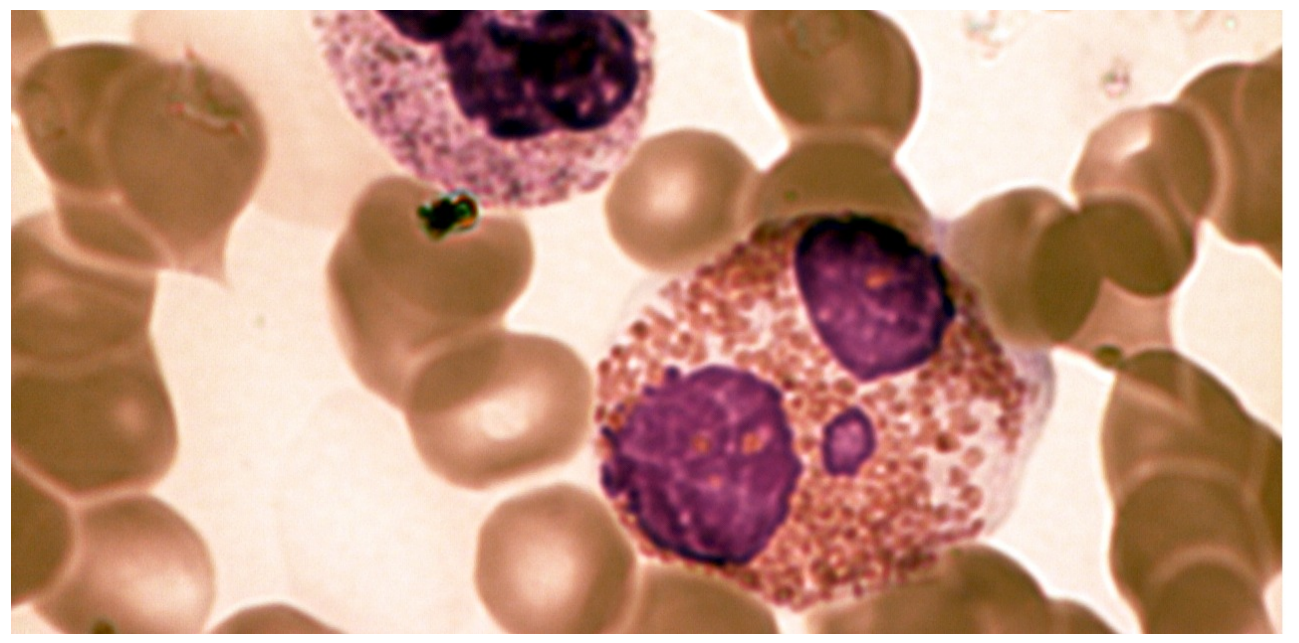

\title{
ASTHMA AND COVID-19: THE EOSINOPHILIC LINK
}

\author{
Carlos Rodriguez ${ }^{1}$, Carlos Veciana ${ }^{2}$ \\ 1 Fundación Jiménez Díaz \\ 2 Hospital Universitario 12 de Octubre
}

\section{Abstract}

The Covid-19 disease caused by the coronavirus SARS-Cov2 has reached pandemic proportions in an extremely short period of time. It happened so fast that, at this point, with more than 200.000 deaths worldwide, and despite the overwhelming efforts enlisted by the global scientific community, we still don't know much about a number of key issues, such as the way the virus causes such a damage in the lungs of patients at risk, how the infection raises the immune response (if any) against it, or what is the long term outcome of patients surviving the devastating complications of the disease. Among the questions that don't seem to gather as much interest, however, one has attired our attention. We are especially keen to find epidemiological factors that could protect people from having the disease, and, at any rate, from presenting the lethal complication thereof: the SARS or Severe Acute Respiratory Syndrome. On this route, after going through the published patient series, we have begun to work on the hypothesis that asthma could be a protective factor in the context of Covid-19.

CONCLUSION: In this paper we analyzed different aspects of previous research on 
asthma, asthma immunology, SARS, and the innate immune system and have come to a starting model of how the asthmatic immune signature (the activation of the Th2 lymphocyte pathway and its main effector cell type, the eosinophil cell) could influence and modulate the response to the Covid-19 infection. In this model, both the Th2 program, with the eosinophil cell at its core, is posited as a natural counterbalance of the Th proinflammatory response (Th1 and Th17). The model proposes a pivotal place for the innate immunity, in which the Pattern Recognition Receptors (particularly the ssRNA recognizer, TLR7) would play a leading role in the rampant inflammatory response to the virus (Th1/Th17 driven) that underlies the development of SARS. IMPORT ANCE: Although not presented as such in the literature, we put forward a vision in which innate immune receptors such as TLR7 and the TH2 programs could be the antagonic forces deciding the fate of lung diseases such as ARDS, asthma or COPD. The careful mining of this model as we describe it herein, could hopefully lead to new urgently needed therapies for the Covid-19 disease.

\section{Introduction}

In December 2019, pneumonia cases caused by a new coronavirus (SARS-cov2) were starting to be diagnosed in Wuhan, China ${ }^{[1][2][3]}$. Since then, the virus has spread to mainland China and all over the world, reaching pandemic proportions.

The SARS-Cov2 virus is a close relative of the SARS-cov1 virus that caused the SARS epidemic in 2003. It belongs to the family of beta-coronavirus, single stranded +RNA viruses, that includes the SARS-Cov1 and the NL63, the latter being a frequent cause of seasonal common cold. Among the viral proteins, the spike (S1) protein is essential for host recognition and viral entry (infection) into the cells. It has been recognized that the cell surface receptor for the S1 protein of SARS-Cov2 is the same as for the SARS-cov1, the virus that caused the 2003 SARS pandemic ${ }^{[4]}$.

In the majority of infected people, the SARS-cov2 virus causes a flu-like disease that resolves spontaneously (the so-called covid-19 disease). In a percentage of cases, however (between 10-20\%, the subg roup of patients at risk of developing lethal complications), after the flu like syndrome, the disease can progress into a Severe Respiratory Distress Syndrome (SARS), which is the cause of hospitalization and eventually of death. Patients at risk of developing SARS after infection with the SARSCov2 virus include older patients ( $>65$ years old), people with one or more comorbidities (such as cardiovascular diseases, high blood pressure, or diabetes) and people 
institutionalized in nursing homes ${ }^{[5]}$.

AN EXCESSIVE INFLAMMAT ORY REACTION IN THE AIRWAYS IS THE CAUSE OF DEATH in COVID-19 PATIENTS.

SARS occurs in the subgroup of patients at risk, where a hyper-inflammatory reaction within the lung airway and in the lung interstitial milieu occurs during the course of the viral infection. This aberrant inflammatory response leads to tissue injury, leaking of fluid into the alveolar cavity, impaired gas exchange, and, eventually, death by respiratory failure.

In a previous paper, we have postulated that the common theme in patients at risk of developing SARS is the background pre-activation of the Renin Angiotensin System (RAS). In these patients, an already turned-on RAS will act as a jump-start for the magnified response of the innate immune system to the SARS-Cov2 viral infection that is witnessed in cases with a severe disease presentation. Based on this premise, we have proposed a treatment plan for the consideration of the scientific and medical community. This treatment is, with small modifications, basically the same as in the so-called SMART asthma protocol that uses a combination of an inhaled glucocorticoid (preferentially budesonide) and an inhaled long acting beta-2 adrenergic agonist (LABA) (preferentially formoterol). The main indication of this protocol during asthma attacks is as a bronchodilator, but as we reviewed the literature, we discovered that the combination of Budesonide/formoterol is a potent local immunosuppressant, that is predicted to precisely counter the cytokine/inflammatory cell storm that underlines the first stages of SARS. In this plan, we recommend to begin treating the patients at risk early on in the disease (at the time of presentation of the first flu-like symptoms), thus emphasizing the need to beg in treatment much before the appearance of dyspnea during the second week of the disease. The sudden presentation of dyspnea is usually the confirmation that SARS is progressing and, oftentimes, too late to take effective therapeutic action against the disease ${ }^{[6]}$.

\section{AST HMA POST ULATES ITSELF AS A POSSIBLE INDEPENDENT PROTECTIVE FACT OR FOR COVID-19}

From the study of all the major multi-center patient series of covid-19 patients (from China ${ }^{[7][8]}$, or the USA ${ }^{[9]}$ ), a few points stand out as interesting possible therapeutic leading points: 1) Among the patients with a severe clinical presentation, there is an overrepresentation of older people as well as of a number of comorbidities (cardiovascular 
diseases, cardiac arrhythmias, high blood pressure, or diabetes). These comorbidities have something in common: an overactivation of the Renin Ang iotensin System (RAS) and a chronic inflammatory basal state. 2) Viral replication rates are essentially the same across sex, age groups and disease severity, indicating that anti-viral drugs are probably not at the core of future anti-Covid-19 therapies. 3) Blood indicators of inflammation as well as lung tissue histopathology evidence the presence of an overwhelming local inflammatory process originating in the bronchoalveolar space and expanding into the lung interstitial milieu. 4) Finally, among all the series of covid-19 (mild or severe presentations alike), there is a striking absence of patients with chronic asthma or with other common related diseases such as allergic rhinitis or atopia ${ }^{[10]}$. Even more telling to the latter point, a recent study from Italy in 23.188 patients dying from Covid-19 disease, among the 16 possible comorbidities cited in the paper, there is no mention of asthma or allergic rhinitis, in spite of the high prevalence of those conditions in the general population ${ }^{[11]}$. This raises the issue of whether suffering from asthma is in itself a protective factor against developing a symptomatic Covid -19 disease and, more especially, against presenting the lethal complications of the disease. This alone goes beyond asthma protecting patients from developing a symptomatic covid-19 disease but, more importantly, from dying from the infection.

\section{ASTHMA AND SARS: THE EOSINOPHIL CONNECTION}

Bronchial asthma is a chronic inflammatory disorder of the airways that affects 300 million people worldwide. It is characterized by bronchial hyperreactivity (BHR), mucus overproduction, and airway remodeling and reversible airway obstruction.

The traditional asthma phenotype is characterized by increased levels of type 2 inflammation mainly mediated by mast cells, eosinophils, basophils, T-helper 2 cells, group 2 innate lymphoid cells (ILC2s), and immunoglobulin E (IgE)-producing B cells ${ }^{[12]}$. Patients with T2 asthma have eosinophilia and other signs of type 2 inflammation including high levels of IL-4, IL-5 and IL-13. Most patients with asthma have different degrees of $\mathrm{T} 2$ hyperactivation.

The term T2 refers thus to a type of immune response. In fact, the type 1 and type 2 immune response classic model redirects to the different immune responses that are regulated by subpopulations of CD4+ T cells known as T helper 1 (TH1) and TH2 cells, respectively. 
TH1 cells secrete interleukin-2 (IL-2), interferon-Y (IFNY) and lymphotoxin- $\alpha$, and stimulate type 1 immunity, which is characterized by prominent phagocytic activity ${ }^{[13]}$, whereas TH2 cells mainly secrete the prototypical cytokines IL-4, IL-5 and IL-13, and stimulate type 2 immunity, which is characterized by high antibody titres and eosinophilia.

Eosinophils are special polymorphonuclear leukocytes. They develop in the bone marrow and migrate into blood, making up about 1-6\% of white blood cells. They were first observed by Wharton Jones in 1846 and named by Paul Ehrlich in 1879, because they contain intracellular granules that are intensely stained by the acidophilic dye eosin.

The presence of large specific granules, also known as secondary granules, is a characteristic feature that disting uishes eosinophils from other granulocytes (neutrophils and basophils). The predominant substances in these granules are four proteins: the antiparasitic (helmintho-toxic) major basic protein (MBP), the bactericidal eosinophil peroxidase (EPO), the eosinophil cationic protein (ECP), and the eosinophil-derived neurotoxin (EDN) ${ }^{[14]}$. The latter two proteins are members of the emerging RNase $A$ gene superfamily, and are capable of degrading single-stranded RNA (sSRNA) substrates with high efficiency ${ }^{[15]}$. The fact that two of the four most abundant eosinophilic specific proteins are ssRNAses has a central role in the physiopathogenesis of asthma but also, as we will see later on, it could have an important bearing in the possible physiopathological mechanisms underlying the protective role of asthma against SARS ${ }^{[16]}$.

Early on in the covid-19 pandemic, there was an observation being made in all patient's series: Eosinopenia or low blood eosinophil count (defined as a reduction of circulating eosinophils <0.01 × 109/l) was frequently present in hospitalized covid-19 patients and, more importantly, it correlated with the severity of the disease. First, in a series of PCRconfirmed SARS-CoV-2 positive patients ( $n=52$ ) and SARS-CoV-2 negative patients ( $n=53$ ) the rate of eosinopenia (decreased number of eosinophils) in SARS-CoV-2 positive patients (79\%) was much higher than that in SARS-CoV-2 negative patients (36\%) (A simple laboratory parameter facilitates early identification of COVID-19 patients). Second, 10 out of 13 patients (77\%) had a reduced eosinophil count of 0.0 (Challenges for NHS hospitals during covid-19 epidemic), or Seven over ten patients presented with eosinophil cytopenia $^{[17]}$. More importantly, eosinopenia was associated with a poor prognosis ${ }^{[18]}$. 
characterized by a massive migration of inflammatory cells and the release of cytokines in the lung. Indeed, a decrease of the eosinophil blood count has been consistently observed in a number of different disease settings, all of them with a common theme: inflammation. Thus, a rapid and persistent decrease in the numbers of circulating eosinophils is a distinctive aspect of the physiological response to acute inflammation ${ }^{[19]}$. Also, eosinopenia (low eosinophil blood count) is an independent predictor of death in patients with pneumonia but not with chronic respiratory disease ${ }^{[20][21]}$. In fact, in another study, a cutoff of $<220$ eosinophils/mm3 was $100 \%$ sensitive for sepsis prediction in critically ill patients ${ }^{[22]}$.

Although important, eosinopenia would only be a good marker of disease severity in processes underlying an inflammatory activity. However, we think there is much more to it than a simple correlation with disease severity in inflammation. We believe that the peripheral blood low count (sometimes straight absence) of eosinophils reflects something very relevant to the physiopathology of inflammation at large, and more concretely to the ARDS that occurs during the course of Covid 19. To put this in the right context, we should recall a few relevant observations from the literature.

1. Samples from lung biopsies or bronchoalveolar aspirates of covid-19 SARS patients show a massive infiltration of inflammatory cells (notably FCN1 macrophages) in a proteinaceous magma, but there is a striking absence of eosinophils in SARS-Cov or in Covid-19 samples $^{[23]}$.

2. The analysis of both the immune cell and cytokine profile in the lungs of SARS indicates a very strong skewing of the immune response towards a Th1 phenotype of inflammation[24] [25].

3. Both asthma and chronic obstructive pulmonary disease (COPD) are characterized by an inflammatory process. However, in asthma, the most striking feature is the eosinophilic infiltration, whereas, in COPD, it is the CD8 T-lymphocytic infiltration of the airway wall[26].

4. In patients with Chronic Obstructive Pulmonary Disease (COPD), a predominant Th1 inflammatory condition, a number of studies have shown that the higher the blood eosinophil count, the greater the positive response to inhaled corticosteroids in the form of reduced exacerbation [27] [28]. suggesting either a mere correlation or a causal effect of the eosinophil rise on the clinical improvement in those patients.

5. Eosinophilia in the airways is a feature of rhinitic patients (even without asthma) ${ }^{[29]}$ and allergic rhinitis is also underrepresented in patients with covid- 19 . 
So far, we have shown that the massive immune response that is behind SARS is predominantly of the proinflammatory type (mainly Th1 but possible others like Th17 too), while signs of even a minimal activation of the Th2 type seem to be absent in SARS. This includes, the lack of presence of the major cellular effector Th2 activation: the eosinophil. It is thus reasonable that both the absence of eosinophils in lung histological samples of covid-19 patients, and the low eosinophil counts in peripheral blood, together with the massive presence of eosinophils in "low risk for SARS" conditions such as asthma and rhinitis, could suggest a protective role of these cells against the covid-19 disease and its complications. In addition, we could hypothesize that activated eosinophils as present in asthma, at least, through the massive production of ssRNA ribonucleases could hamper viral infectivity of SARS-cov2, whose genome, as any of the members of the coronavirus family, consists of a single chain of ssRNA.

\section{THE INNATE IMMUNE SYSTEM IN SARS. THE TLR7 CONNECTION}

The innate immune system is the first line of defense at the surface of the airway epithelium. There, there is a group of molecules (both at the cell surface and inside the cells) that are in charge of recognizing and eliminating foreing organisms.

The pattern recognition receptor in charge of identifying and countering infectious RNA viruses like the SARS-Cov19, the TLR7, is a member of the Toll Like Receptor superfamily of PRR. As other family members (TLR3, 7, 8, and 9), it is an endolysosomal transmembrane protein that encounters the virus within that compartment. TLR7 is expressed in bronchial epithelial cells, airway smooth muscle and several immune cell types, including lung plasmacytoid dendritic cells, natural killer cells (NKT), B lymphocytes and eosinophil[30] [31]. In nature, TLR7 recognizes ssRNA sequences with high G-U content, as present in most RNA viruses [32][33][34][35]. Upon activation, the TLR7 receptor orchestrates a complex anti-viral response, including the secretion of the antiviral IF IS and compounded by a broader pro-inflammatory response mediated by de secretion of Il-1 beta and gamma interferon and a number of other cytokines, all together finally resulting in the proliferation and activation of $\mathrm{T}, \mathrm{B}$ lymphocytes and the final effector cells, neutrophils and macrophages. This inflammatory response needs to be closely modulated so that it can lead to the efficient clearance of the invading virus, while maintaining the proper tissue homeostasis (notably avoiding unwanted tissue damage).

Although not yet thoroughly studied in the context of the SARS-Cov2 pandemic, different 
previous observations point to a key role of TLR7 in SARS-Cov2. First, TLR7 appears to be the key receptor for the first line response to RNA viruses ${ }^{[36]}$. More to the point, SARS-CoV ssRNAs had powerful immunostimulatory activities to induce considerable level of pro-inflammatory cytokine TNF-a, IL-6 and IL-12 release via the TLR7 and TLR8, almost 2-fold higher than the strong stimulatory ssRNA40 that was found previously from other virus ${ }^{[37]}$. Moreover, SARS-CoV ssRNA was able to cause acute lung injury in mice with a high mortality rate in vivo, suggesting that SARS-CoV specific GU-rich ssRNA plays a very important role in the cytokine storm associated with a dysregulation of the innate immunity and could open a new therapeutic strategy ${ }^{[38]}$. Finally, ssRNA40 induces cell death in mice through binding to TLR7 and activation of downstream signaling molecules, while TLR7-/- mice are entirely protected against sSRNA40 induced cell death $^{[39]}$.

\section{TLR7 ACTIVATION CANCELS OUT THE TH2 PHENOTYPE}

There are good reasons to view the TLR7 receptor as a counterbalance of the Th2 axis. First, TLR7 activation prevents ovalbumin-induced airway hyperreactivity, eosinophilic inflammation, goblet cell hyperplasia and airway remodeling in murine models of asthma. Second, TLR7 activation also inhibits viral replication in the lung and prevents virusinduced airway hyperreactivity. Furthermore, it has recently been shown that stimulating TLR7 rapidly relaxes airway smooth muscle, dilating the airways ${ }^{[40]}$. The abrogation of the Th2 pathway by TLR7 is so relevant that it has recently been exploited to the design of novel anti-asthmatic compounds with TLR7 agonistic activity ${ }^{[41]}$.

By contrast, Th2-inducing pathological conditions such as parasitic diseases alter the response to viral infections through yet unclear mechanisms. On the one hand, IL-4, the mastermind of theTh2 response, inhibits the secretion of antiviral type I interferons and, on the other hand, it suppresses TLR7-induced production of pro-inflammatory cytokines such as TNF $\alpha$, IL-12p70 and IL- 6 by inhibiting $\gamma$ IFN-dependent and NFkB-dependent responses $^{[42]}$

Taken together this shows that the signaling pathway activated by the putative pattern recognition receptor of SARS-Cov2, the TLR7, and the Th2 pathway that is activated in asthma are, in fact, antagonic. We think that the existence of this counterregulatory loop could provide for a mechanistic hypothesis for the physiopathology of the first steps in the covid-19 disease and, central to our thesis, an answer to the observation that people with asthma and other related diseases could be protected from the dire complications 
of the covid-19 disease. This could translate into possible new therapies for SARS/Covid19. One could easily imagine that TLR7 antagonists would tilt the balance towards the activation of the Th2 axis, therefore stopping the self-aggression and lung damage as seen in SARS. Also, Th2 activators, either proteins (like interleukin-25 or interleukin-33) or small molecules would be well suited to be used as first line therapy in non-asthmatic patients at risk.

This leaves us with many open questions/speculations that can be inferred from such a thesis. For instance, there is rapidly growing evidence that cigarette smoking is underrepresented in covid-19 patients. This observation has lead to the claim that, as much contrary to common sense that it might sound, current cigarette smoking might protect smokers from the lethal complications of this disease ${ }^{[43][44]}$. Besides the different patient series that unambiguously show the low prevalence of current smokers among patients with severe forms of SARS, evidence from the asthma literature also establishes a common thread between cigarette smoke and our thesis. In fact, it has been shown that short cigarette smoke exposure is sufficient to facilitate allergic sensitisation and the development of low-dose HDM-induced allergic asthma, possibly by affecting dendritic cell function and skewed Th2 lymphocyte differentiation ${ }^{[45]}$. This alone could suffice to reinforce the argument in favor of the hypothetical protective effect of cigarette smoking could be mediated by the activation of the Th2-eosinophil axis. Contrary to the opinions suggesting that we should not vent this kind of speculations, we think that, as scientists, we should just confront the facts, check them up for scientific truthfulness, and try to draw conclusions from them. In this particular case, it would be interesting to analyse, as has already been proposed, what would be the role of nicotine or any other ingredient in cigarette smoke mediating this effect. This could lead to much needed novel therapies for the disease.

Finally, we could also speculate that the high prevalence of parasitic diseases and therefore the pervasiveness of (blood or tissue) eosinophilia and the relatively low incidence of the pandemic in areas like Tropical Africa ${ }^{[46]}$ or the Indian subcontinent ${ }^{[47][48][49]}$, could be somehow linked. Only time and further research will tell us if there is a relevant connection here.

At this point in time, because of the massive amount of information and scientific endeavour being deployed in search for answers and treatments, we cannot afford to close any open question, as long as there is a minimal likelihood that it could lead us to 
some new therapeutic avenue for the covi-19 pandemic. We have shown here that there are strong reasons to believe that the asthma/eosinophil connection could lead to new therapeutic avenues for the covid-19 disease and for the group lethal inflammatory conditions at large.

References

1. ^ Chaolin Huang, Yeming Wang, Xingwang Li, Lili Ren, Jianping Zhao, Yi Hu. (2020). Clinical features of patients infected with 2019 novel coronavirus in Wuhan, China. The Lancet, vol. 395 (10223), 497-506. doi:10.1016/s0140-6736(20)30183-5.

2. `Chen Wang, Peter W Horby, Frederick G Hayden, George F Gao. (2020). A novel coronavirus outbreak of global health concern. The Lancet, vol. 395 (10223), 470-473. doi:10.1016/s0140-6736(20)30185-9.

3. `Na Zhu, Dingyu Zhang, Wenling Wang, Xingwang Li, Bo Yang, Jingdong Song. (2020). A Novel Coronavirus from Patients with Pneumonia in China, 2019. N EnglJ Med, vol. 382 (8), 727-733. doi:10.1056/nejmoa2001017.

4. ^ Kristian G. Andersen, Andrew Rambaut, W. Ian Lipkin, Edward C. Holmes, Robert F. Garry. (2020). The proximal origin of SARS-CoV-2. Nat Med, vol. 26 (4), 450-452. doi:10.1038/s41591-020-0820-9.

5. 'Damian N Valencia. (2020). Brief Review on COVID-19: The 2020 Pandemic Caused by SARS-CoV-2. doi:10.7759/cureus.7386.

6. ^ Rodriguez, Carlos; Veciana, Carlos. (2020). The combination of inhaled budesonide and formoterol as an early treatment for the covid-19 disease..

7. ^ Wei-jie Guan, Wen-hua Liang, Yi Zhao, Heng-rui Liang, Zi-sheng Chen, Yi-min Li. (2020). Comorbidity and its impact on 1590 patients with Covid-19 in China: A Nationwide Analysis. Eur RespirJ. doi:10.1183/13993003.00547-2020.

8. ^ Wei-jie Guan, Zheng-yi Ni, Yu Hu, Wen-hua Liang, Chun-quan Ou, Jian-xing He. (2020). Clinical Characteristics of Coronavirus Disease 2019 in China. N EnglJ Med. doi:10.1056/nejmoa2002032.

9. ^ CDC COVID-19 Response Team, Nancy Chow, Katherine Fleming-Dutra, Ryan Gierke, Aron Hall, Michelle Hughes. (2020). Preliminary Estimates of the Prevalence of Selected Underlying Health Conditions Among Patients with Coronavirus Disease 2019 - United States, February 12-March 28, 2020. MMWR Morb. Mortal. Wkly. Rep., vol. 69 (13), 382-386. doi:10.15585/mmwr.mm6913e2.

10. ^ Jin-jin Zhang, Xiang Dong, Yi-yuan Cao, Ya-dong Yuan, Yi-bin Yang, You-qin Yan. (2020). Clinical characteristics of 140 patients infected with SARS-COV-2 in Wuhan, China. Allergy. doi:10.1111/all.14238. 
11. ^ (2020). Characteristics of SARS-COV-2 patients dying in Italy Report based on available data on April 23th, 2020. Instituto Superiore di Sanitá.

12. ^ John V. Fahy. (2014). Type 2 inflammation in asthma - present in most, absent in many. Nat Rev Immunol, vol. 15 (1), 57-65. doi:10.1038/nri3786.

13. ^ B. Spellberg, J. E. Edwards. (2001). Type 1/Type 2 Immunity in Infectious Diseases. Clinical Infectious Diseases, vol. 32 (1), 76-102. doi:10.1086/317537.

14. ^Claire N. McBrien, Andrew Menzies-Gow. (2017). The Biology of Eosinophils and Their Role in Asthma. Front. Med., vol. 4 . doi:10.3389/fmed.2017.00093.

15. ^ Lu Lu, Jiarui Li, Mohammed Moussaoui, Ester Boix. (2018). Immune Modulation by Human Secreted RNases at the Extracellular Space. Front. Immunol., vol. 9. doi:10.3389/fimmu.2018.01012.

16. ^ Giancarlo Marone, Francescopaolo Granata, Valentina Pucino, Antonio Pecoraro, Enrico Heffler, Stefania Loffredo. (2019). The Intriguing Role of Interleukin 13 in the Pathophysiology of Asthma. Front. Pharmacol., vol. 10. doi:10.3389/fphar.2019.01387.

17. ^ LiYX1, WuW1, Yang T2, Zhou W1, Fu YM1, Feng QM1, Ye JM1.. (2020). Characteristics of peripheral blood leukocyte differential counts in patients with COVID-19. ChinJ Intern Med, vol. 59.

18. ` Yingzhen Du, Lei Tu, Pingjun Zhu, Mi Mu, Runsheng Wang, Pengcheng Yang. (2020). Clinical Features of 85 Fatal Cases of COVID-19 from Wuhan: A Retrospective Observational Study. Am J Respir Crit Care Med. doi:10.1164/rccm.202003-0543oc.

19. ^ David A. Bass, Thomas A. Gonwa, Pamela Szejda, M. Susan Cousart, Lawrence R. DeChatelet, Charles E. McCall. (1980). Eosinopenia of Acute Infection.J. Clin. Invest., vol. 65 (6), 1265-1271. doi:10.1172/jci109789.

20. ^ Carlos Echevarria et Al. (2014). 30 day mortality and eosinopenia in patients with pneumonia. European Respiratory Journal, vol. 44 .

21. `Jin-jin Zhang, Xiang Dong, Yi-yuan Cao, Ya-dong Yuan, Yi-bin Yang, You-qin Yan. (2020). Clinical characteristics of 140 patients infected with SARS-CoV-2 in Wuhan, China. Allergy. doi:10.1111/all.14238.

22. ^A Savitskiy, V Rudnov, V Bagin. (2015). Eosinopenia as a marker of sepsis and mortality in critically ill patients. Critical Care, vol. 19 (Supp/ 1), P47. doi:10.1186/cc14127.

23. ^ Minfeng Liao, Yang Liu, Jin Yuan, Yanling Wen, Gang Xu, Juanjuan Zhao. (2020). The landscape of lung bronchoalveolar immune cells in COVID-19 revealed by single-cell RNA sequencing. doi:10.1101/2020.02.23.20026690. 
24. ` Li Liu, Qiang Wei, Qingqing Lin, Jun Fang, Haibo Wang, Hauyee Kwok. (2019). Antispike IgG causes severe acute lung injury by skewing macrophage responses during acute SARS-CoV infection. doi:10.1172/jci.insight.123158.

25. ^ Conti P et Al. (2020). Induction of pro-inflammatory cytokines (IL-1 and IL-6) and lung inflammation by Coronavirus-19 (COVI-19 or SARS-COV-2): anti-inflammatory strategies. J Biol Regul Homeost Agents.

26. ^ M. Saetta, G. Turato. (2001). Airway pathology in asthma. doi:10.1183/09031936.01.00229501.

27. ^ Salman H. Siddiqui, Alessandro Guasconi, Jørgen Vestbo, PaulJones, Alvar Agusti, Pierluigi Paggiaro. (2015). Blood Eosinophils: A Biomarker of Response to Extrafine Beclomethasone/Formoterol in Chronic Obstructive Pulmonary Disease. Am J Respir Crit Care Med, vol. 192 (4), 523-525. doi:10.1164/rccm.201502-0235/e.

28. ^ Dave Singh, Umme Kolsum, Chris E. Brightling, Nicholas Locantore, Alvar Agusti, Ruth Tal-Singer. (2014). Eosinophilic inflammation in COPD: prevalence and clinical characteristics. Eur RespirJ, vol. 44 (6), 1697-1700. doi:10.1183/09031936.00162414.

29. ${ }^{\wedge}, \ldots, \ldots$. (2000). Eosinophils are a feature of upper and lower airway pathology in nonatopic asthma, irrespective of the presence of rhinitis. Clin Exp Allergy, vol. 30 (5), 663669. doi:10.1046/j.1365-2222.2000.00804.x.

30. ` Julien Cherfils-Vicini, Sophia Platonova, Mélanie Gillard, Ludivine Laurans, Pierre Validire, Rafaele Caliandro. (2010). Triggering of TLR7 and TLR8 expressed by human lung cancer cells induces cell survival and chemoresistance. J. Clin. Invest., vol. 120 (4), 1285-1297. doi:10.1172/jci36551.

31. ^ Anna-Karin Ekman, Mikael Adner, Lars-Olaf Cardell. (2011). Toll-like receptor 7 activation reduces the contractile response of airway smooth muscle. European Journal of Pharmacology, vol. 652 (1-3), 145-151. doi:10.1016/j.ejphar.2010.11.009.

32. ^ Canrong Wu, Yang Liu, Yueying Yang, Peng Zhang, Wu Zhong, Yali Wang. (2020). Analysis of therapeutic targets for SARS-COV-2 and discovery of potential drugs by computational methods. Acta Pharmaceutica Sinica B. doi:10.1016/j.apsb.2020.02.008.

33. ^ S. S. Diebold. (2004). Innate Antiviral Responses by Means of TLR7-Mediated Recognition of Single-Stranded RNA. Science, vol. 303 (5663), 1529-1531. doi:10.1126/science.1093616.

34. ^ Michael P. Gantier, Stephen Tong, Mark A. Behlke, Dakang Xu, Simon Phipps, PaulS. Foster. (2008). TLR7 Is Involved in Sequence-Specific Sensing of Single-Stranded RNAS in Human Macrophages. J Immunol, vol. 180 (4), 2117-2124. 
doi:10.4049/jimmunol.180.4.2117.

35. ^ Alexander H. Dalpke, Mark Helm. (2012). RNA mediated toll-like receptor stimulation in health and disease. RNA Biology, vol. 9 (6), 828-842. doi:10.4161/rna.20206.

36. ` S. Stegemann-Koniszewski, Andreas Jeron, Marcus Gereke, Robert Geffers, Andrea Kröger, Matthias Gunzer. (2016). Alveolar Type II Epithelial Cells Contribute to the AntiInfluenza A Virus Response in the Lung by Integrating Pathogen- and Microenvironment-Derived Signals. mBio, vol. 7 (3). doi:10.1128/mbio.00276-16.

37. ` Yan Li, Ming Chen, Hongwei Cao, Yuanfeng Zhu, Jiang Zheng, Hong Zhou. (2013). Extraordinary GU-rich single-strand RNA identified from SARS coronavirus contributes an excessive innate immune response. Microbes and Infection, vol. 15 (2), 88-95. doi:10.1016/j.micinf.2012.10.008.

38. ^ Dale L Barnard, Yohichi Kumaki. (2011). Recent developments in anti-severe acute respiratory syndrome coronavirus chemotherapy. Future Virology, vol. 6 (5), 615-631. doi:10.2217/fvl.11.33.

39. ^Sabrina M. Lehmann, Karen Rosenberger, Christina Krüger, Piet Habbel, Katja Derkow, David Kaul. (2012). Extracellularly Delivered Single-Stranded Viral RNA Causes Neurodegeneration Dependent on TLR7. J.I., vol. 189 (3), 1448-1458. doi:10.4049/jimmunol.1201078.

40. ^ Matthew G. Drake, Elad H. Kaufman, Allison D. Fryer, David B. Jacoby. (2012). The Therapeutic Potential of Toll-Like Receptor 7 Stimulation in Asthma. IADT, vol. 11 (6), 484-491. doi:10.2174/187152812803589967.

41. ^ John Bell, Mike Dymond, Mark Biffen, Stephen Delaney, David Keeling, Hui Zhang. (2018). Temporal cytokine and lymphoid responses to an inhaled TLR7 antedrug agonist in the cynomolgus monkey demonstrates potential safety and tolerability of this approach. Toxicology and Applied Pharmacology, vol. 338, 9-19. doi:10.1016/j.taap.2017.11.002.

42. ^ Uma Sriram, Jun Xu, Robert W. Chain, Linda Varghese, Marita Chakhtoura, Heather L. Bennett. (2014). IL-4 Suppresses the Responses to TLR7 and TLR9 Stimulation and Increases the Permissiveness to Retroviral Infection of Murine Conventional Dendritic Cells. PLoS ONE, vol. 9 (1), e87668. doi:10.1371/journal.pone.0087668.

43. ^ Konstantinos Farsalinos, Anastasia Barbouni, Raymond Niaura. (2020). Smoking, vaping and hospitalization for COVID-19. Qeios. doi:10.32388/26908a.10.

44. ^ Makoto Miyara, Florence Tubach, Valérie POURCHER, Capucine Morelot-Panzini, Julie Pernet, Julien Haroche. (2020). Low incidence of daily active tobacco smoking in patients with symptomatic COVID-19. Qeios. doi:10.32388/wpp19w.3. 
45. ^ Ellen A. Lanckacker, Kurt G. Tournoy, Hamida Hammad, Gabriele Holtappels, Bart N. Lambrecht, Guy F. Joos. (2012). Short cigarette smoke exposure facilitates sensitisation and asthma development in mice. Eur Respir J, vol. 41 (5), 1189-1199. doi:10.1183/09031936.00096612.

46. ^Ayodele Olushola Ilesanmi, GinnikachiJennifer Ekwe, Rosemary Isioma Ilesanmi, Damilola Temitope Ogundele, Jacob Kehinde Akintunde, Oluwasogo Adewole Olalubi. (2016). Prevalence of latent eosinophilia among occupational gardeners at Babcock University, Nigeria. Asian Pacific Journal of Tropical Biomedicine, vol. 6 (6), 511-515. doi:10.1016/j.apjtb.2015.12.022.

47. ^D Ray, R Abel, K G Selvaraj. (1993). Epidemiology of pulmonary eosinophilia in rural south India--a prospective study, 1981-86.. Journal of Epidemiology \& Community Health, vol. 47 (6), 469-474. doi:10.1136/jech.47.6.469.

48. ^Yogendra Vet al. (2011). Blood and Tissue Eosinophilia: A Study at the MVJ Medical College \& Research Hospital with Review of Literature. RGUHS J Med Sciences, vol. 1.

49. `Neha Thakur, Narendra Rai. (2016). Alarmingly High Incidence of Eosinophilia in Barabanki and Neighboring Districts of Eastern Uttar Pradesh: A Prospective HospitalBased Study. J Trop Pediatr. doi:10.1093/tropej/fmw035. 